\title{
Underground Operator Monitoring Platform Based on Ultra-Wide Band WSN
}

\author{
https://doi.org/10.3991/ijoe.v14i10.9307 \\ Weicai Xie ${ }^{(\varpi)}$ \\ Hunan Institute of Engineering, Xiangtan, China; \\ Hunan Province Cooperative Innovation Center for Wind Power Equipment and Energy Con- \\ version, Xiangtan, China \\ xieweicaiweicai@163.com \\ Xiaofeng $\mathrm{Li}$ \\ Hunan Institute of Engineering, Xiangtan, China; \\ Xi Long \\ PowerChina Zhongnan Engineering Co. Ltd., Changsha, China
}

\begin{abstract}
This paper aims to accurately locate underground personnel in coal mines. For this purpose, an underground personnel positioning platform was established on the wireless sensor network (WSN). Specifically, the ultra-wide band (UWB) and the time difference of arrival (TDOA) positioning algorithm were introduced briefly, in view of the underground operation environment. Then, the underground operator monitoring platform was developed based on UWB-WSN and compared it with different positioning techniques through experiments. The results show that the proposed platform achieved a high positioning accuracy and satisfied the needs of real-time monitoring of underground personnel. The research findings shed new light on the mitigation of personnel and property losses in coal mine accidents.
\end{abstract}

Keywords-Ultra-wide band (UWB), wireless sensor network (WSN), underground positioning

\section{$1 \quad$ Introduction}

The exploitation of mineral resources mostly takes place deep underground. As a result, the operators often face the risks of exposure to harmful gases (e.g. CH4 and $\mathrm{CO}$ ), high temperature, tunnel collapse, etc. $[1,2]$ Once an accident occurs, it is difficult for rescuers to identify the exact location and actual condition of the trapped operators. Thus, the precise positioning of operators is essential to the work safety of underground exploitation of mineral resources [3].

Much research has been done on underground personnel positioning. For instance, Derby (1991), a South African company, developed an automated vehicle positioning system based on infrared communication [4]. The Mount Isa Mineral Company of 
Australia (1995) invented a personnel positioning system based on radio-frequency identification (RFID) to prevent operators from entering high-risk areas [5]. South Africa (2005) began to apply the RFID to the mining industry and achieved remarkable results: this technique enabled managers to track and locate equipment and employees [6]. Yikeya (2006) pioneered the use of Wi-Fi to track and locate underground operators [7].

The underground exploitation of mineral resources features complex terrains, long and narrow roadways, and fluctuating humidity. These conditions may result in an obvious multipath effect. To ensure the positioning accuracy, the interference in wireless transmission must be considered in the positioning algorithm. However, the traditional positioning techniques have various limitations underground [8]. With the development of communication technology, the ultra-wide band (UWB) wireless communication technique has emerged as a good solution to the positioning of underground operators. The UWB supports power-saving and fast transmission of signals that are difficult to intercept or interfere [9]. What is more, the centimeter level accuracy makes it a popular tool in both military and civilian fields. Thus, this technique has been extensively applied in mobile target positioning, object tracking and personnel positioning [10].

In light of the above, this paper designs an underground personnel positioning platform based on the UWB wireless sensor network (WSN). Specifically, the ultra-wide band (UWB) and the time difference of arrival (TDOA) positioning algorithm were introduced briefly, in view of the underground operation environment. Then, the underground operator monitoring platform was developed based on UWB-WSN with a power-saving tag. Finally, the proposed platform was compared with different positioning techniques through experiments. The results show that the proposed platform achieved a high positioning accuracy and satisfied the needs of real-time monitoring of underground personnel.

\section{UWB Technology}

UWB is a short-range wireless communication technology, whose transmission distance is usually less than $10 \mathrm{~m}$ and working band falls between 3.1 and $10.6 \mathrm{GHz}$. Capable of transmitting ultra-low power signals over a wide spectrum, the UWB can realize the data rate up to hundreds of megabytes. This technology has been widely adopted for precision detection, smart home and indoor communication [12], thanks to its wide spectrum and fast transmission.

Besides the UWB, many other short-range wireless positioning technologies have been developed and investigated. The typical examples include infrared positioning, ultrasonic positioning, RFID positioning, Bluetooth positioning, Wi-Fi positioning and ZigBee positioning [13]. Table 1 compares these technologies in five dimensions. Figure 1 contrasts the spectrum and power of the UWB with those of the other technologies. 
Table 1. Comparison between different short-range wireless positioning technologies

\begin{tabular}{|c|c|c|c|c|c|}
\hline & Accuracy & Penetrability & Anti-interference & Complexity & Cost \\
\hline Infrared & $\bullet \bullet \bullet \bullet \circ$ & 00000 & 00000 & $\bullet \bullet \bullet \bullet \bullet$ & $\bullet \bullet ० ० ०$ \\
\hline Ultrasonic & $\bullet \bullet \bullet \bullet \bullet$ & $\bullet \circ 000$ & $\bullet \bullet \bullet ० ०$ & $\bullet \bullet \circ ० \circ$ & $\bullet \bullet \bullet \bullet \bullet$ \\
\hline RFID & $\bullet \bullet \bullet \bullet \bullet$ & $\bullet \bullet \bullet \circ \circ$ & $\bullet \bullet \circ \circ ০$ & $\bullet \bullet \circ ০ ০$ & $\bullet \bullet \circ ০ ০$ \\
\hline Bluetooth & $\bullet \bullet \bullet \circ ०$ & $\bullet \bullet \bullet \circ ०$ & $\bullet \bullet \circ \circ \circ$ & $\bullet \bullet \bullet \circ \circ$ & $\bullet \bullet \bullet \circ \circ$ \\
\hline $\mathrm{WiFi}$ & $\bullet \circ \circ 0 \circ$ & $\bullet \bullet \bullet \circ \circ$ & $\bullet \bullet \bullet \bullet \bullet$ & $\bullet \circ 000$ & $\bullet \circ 000$ \\
\hline ZigBee & $\bullet \bullet \circ ০ ০$ & $\bullet \bullet \bullet \bullet \circ$ & $\bullet \bullet \bullet \circ ०$ & $\bullet \bullet \circ ০ ০$ & $\bullet \bullet \bullet \circ \circ$ \\
\hline UWB & $\bullet \bullet \bullet \bullet \bullet$ & $\bullet \bullet \bullet \bullet \bullet$ & $\bullet \bullet \bullet \bullet \circ$ & $\bullet \bullet \bullet \circ \circ$ & $\bullet \bullet \bullet \circ \circ$ \\
\hline
\end{tabular}

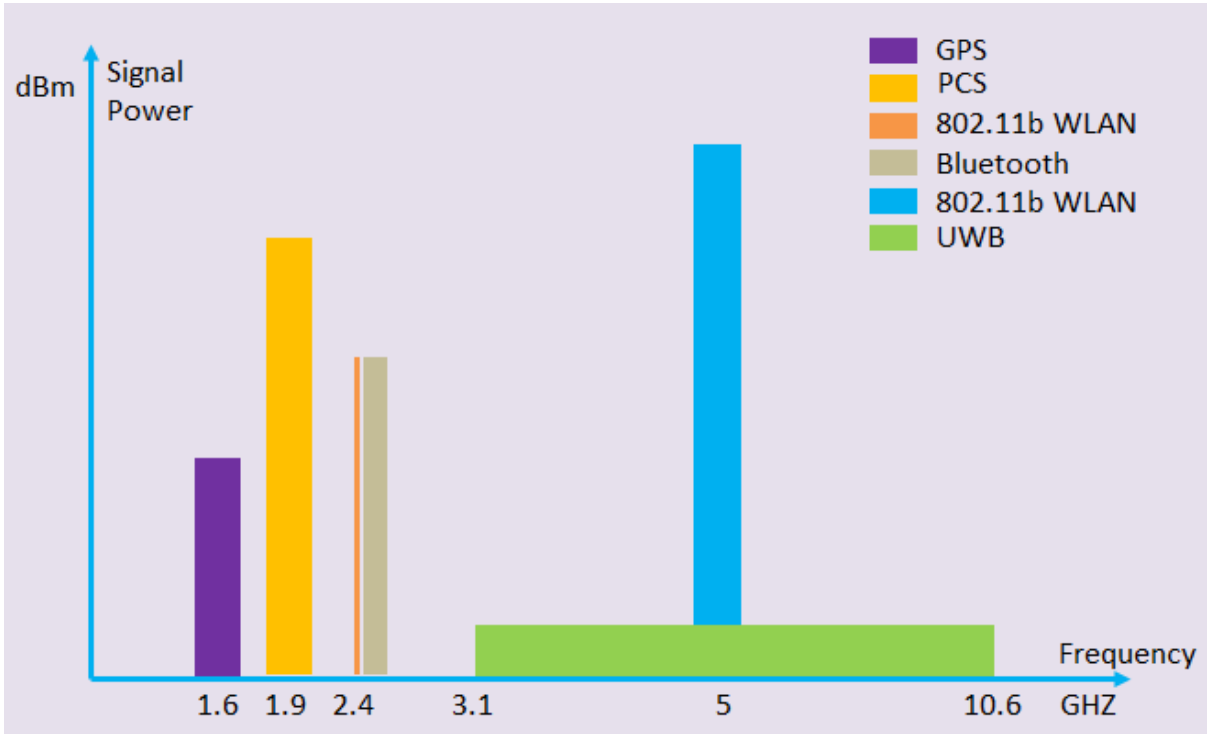

Fig. 1. Spectrum and power of different short-range wireless positioning technologies

As shown in the figure, the UWB enjoys the widest spectrum and the minimum power spectral density among the existing technologies. In general, the UWB is a desirable tool for precise positioning of underground personnel and equipment, due to the following advantages:

1. High accuracy: The positioning accuracy of $10 \mathrm{~cm}$ and high refresh speed can meet the real-time positioning requirements of underground personnel and equipment.

2. Good multipath resistance: With high-resolution signals, the UWB can overcome the multipath fading of signals caused by the rugged walls and complex roadways underground.

3. Strong interference resistance: The communication frequency of UWB is much higher than the frequency of underground noises, and the communication signal has a very wide frequency band. Thus, the UWB boasts a good noise reduction performance. Under the same code speed, the UWB is more immune to interference than IEEE, 802.11a, IEEE, 802.11b and Bluetooth. 
4. Low power consumption: Normally, a wireless communication system has to consume a certain amount of energy to continuously transmit a carrier wave. The UWB replaces the carrier wave with an instantaneous pulse wave as needed. Therefore, it can provide the required standby time for underground communication equipment.

The UWB can be adopted for 1D positioning in tunnels, railways and mines, 2D positioning in factories, warehouses and prisons and $3 \mathrm{D}$ positioning in urban complexes, hospitals and other public places. As shown in Figure 2, the information is captured by the wireless sensors, and sent to the positioning engine to analyse the location of personnel or equipment; finally, the location information is displayed visually on the monitors.

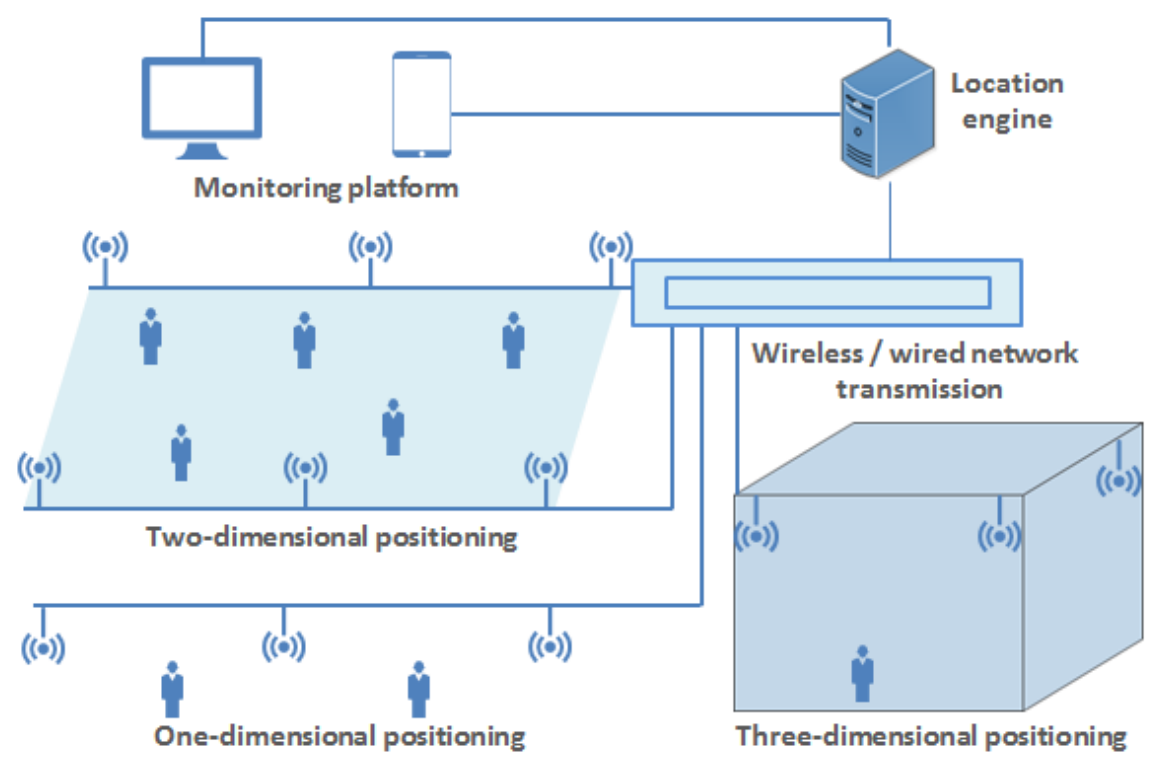

Fig. 2. Structure of UWB-WSN

\section{TDOA Algorithm}

The TDOA is a relatively simple algorithm that only requires strict time synchronization between sensor nodes, eliminating the need for synchronization between sensor nodes and the clock of the mobile nodes. The principle is to locate the wireless emitter based on the time difference of wireless signals arriving at different sensor nodes [1416]. As shown in Figure 3, the specific steps of the TDOA algorithm are as follows.

1. The sensor node sends the data from the same signal measured at the same time to the positioning engine.

2. The positioning engine calculates the time difference between the wireless signals arriving at two sensor nodes. 
3. The time difference is converted into distance difference, and the hyperbolic curve of the difference is plotted.

4. Repeat the above steps for other sensor nodes, and finally obtain multiple hyperbolic curves. The transmitting node can be positioned according to the intersection of several hyperbola.

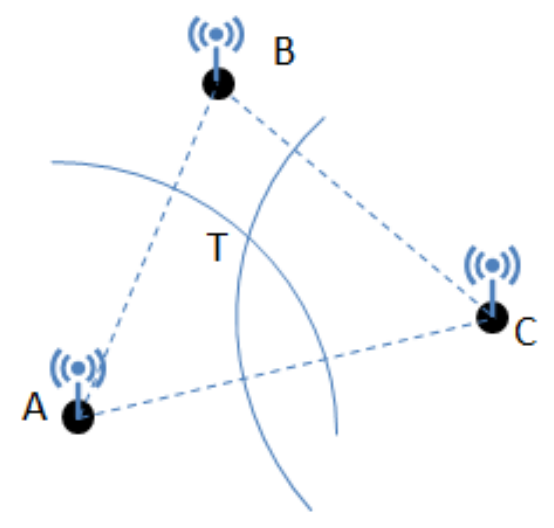

Fig. 3. Workflow of the TDOA

Let $(\mathrm{x}, \mathrm{y})$ be the coordinates of the transmitting node $\mathrm{T}$, and ta, tb and tc be the arrival time from $\mathrm{T}$ to each of the three sensors. Then, the time difference can be obtained by the following formula:

$$
\left\{\begin{array}{l}
T_{a b}=\left|t_{a}-t_{b}\right| \\
T_{a c}=\left|t_{a}-t_{c}\right|
\end{array}\right.
$$

Let constant $\mathrm{c}$ be the speed of the wireless signal. Then, the distance can be calculated as:

$$
\left\{\begin{array}{l}
r_{a b}=c \cdot T_{a b} \\
r_{a c}=c \cdot T_{a c}
\end{array}\right.
$$

Let (xa, ya), (xb, yb) and (xc, yc) be the coordinates of sensors A, B and C, respectively. Then, the following relationships can be established:

$$
\left\{\begin{array}{l}
r_{a b}=\sqrt{\left(x_{a}-x\right)^{2}+\left(y_{a}-y\right)^{2}}-\sqrt{\left(x_{b}-x\right)^{2}+\left(y_{b}-y\right)^{2}} \\
r_{a c}=\sqrt{\left(x_{a}-x\right)^{2}+\left(y_{a}-y\right)^{2}}-\sqrt{\left(x_{c}-x\right)^{2}+\left(y_{c}-y\right)^{2}}
\end{array}\right.
$$

The coordinates of the transmitting node can be derived from the above two equations. 


\section{UWB-WSN Platform}

\subsection{Overall design}

The UWB-WSN monitoring platform for underground operators mainly consists of an application layer, a processing layer, a transport layer and a device layer. The main structure of the platform is shown in Figure 4 below.

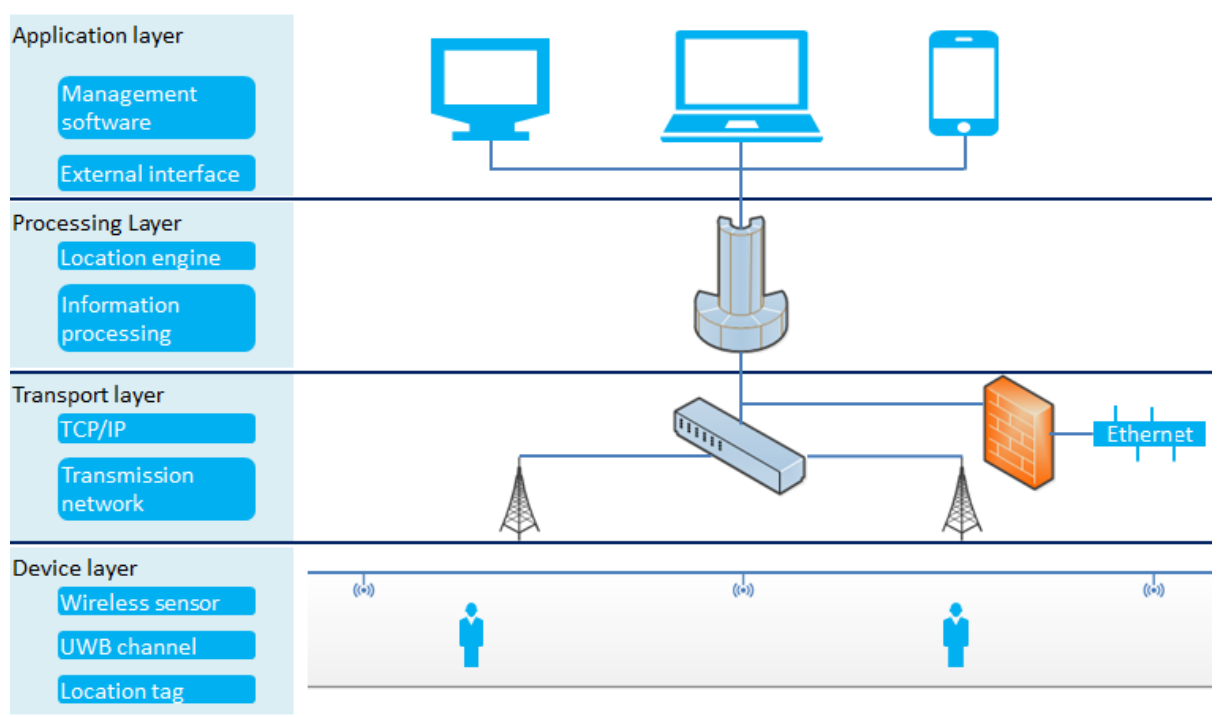

Fig. 4. Structure of the UWB-WSN monitoring platform

The application layer includes management software, monitoring platform and monitoring terminal. The management software maintains the information of operators and equipment, updates the geographic layer, and performs topology, path and buffer analyses. The monitoring platform and terminal mainly support the real-time monitoring and on-site rescue of operators and equipment.

The processing layer emcompasses positioning engine, data processing server and data storage server. The positioning engine can identify the coordinates and identities of operators and equipment through analysis on the signals uploaded by wireless sensors. The data processing server visualizes map information and realizes various other functions. The data storage server stores all the data of the platform, such as the personnel identity, moving trajectories and permission information.e

The transport layer lies between the processing layer and the device layer. The data can be transferred through wired mode or wireless mode or the combination of the two transmission modes. Since the monitoring platform is mainly used within the enterprise, it is only accessible within the enterprise intranet. However, the platform can also be connected to the ethernet if the security authorities want to monitor the security of the enterprise. 
The equipment layer covers wireless sensors, UWB short-range communication network and portable communication tag. The sensors are arranged linearly as the underground working environment is dominated by roadways. The interval between the sensors is usually $50 \mathrm{~m}$, due to short communication distance of the UWB.

\subsection{Tag design}

As shown in Figure 5, tach UWB location tag is composed of microcontroller unit (MCU), battery, audio-visual alarm module, programming interface, wireless transceiver chip, antenna and crystal oscillator.

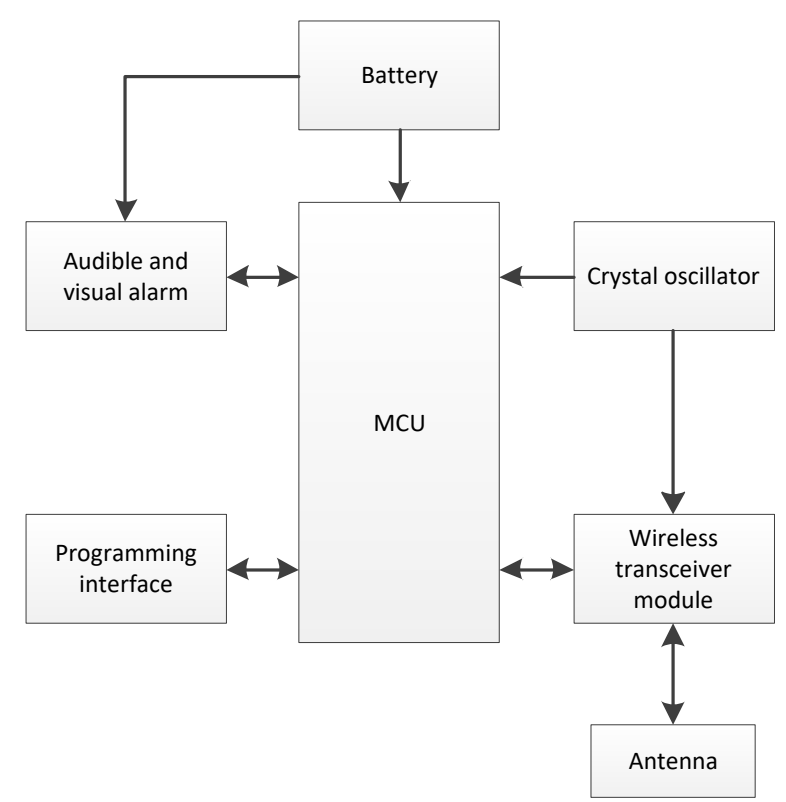

Fig. 5. Hardware structure of location tag

Considering the limited space of underground operation, the location tag must be as small as possible to facilitate carrying. Here, two types of location tags, a bracelet tag and a helmet tag, are designed according to the job contents of the operators. Besides, the size of the MCU was minimized to reduce the power consumption. The workflow of the tag is shown in Figure 6.

Two working modes were designed for the tag, namely, normal mode and low power mode. In the normal mode, the tag sends UWB signals every few seconds, such that the monitoring platform can display the location information of the tag in real time. Since the tag is always emitting signals, it consumes a lot of power in the normal mode. When the battery level falls below a certain threshold, the tag will automatically switch to low power mode. In this mode, the only power consumption takes place at the reception of the wake-up signal and the sending of UWB signal. The low power mode applies to the scenario that does not require the real-time location of the tag. 


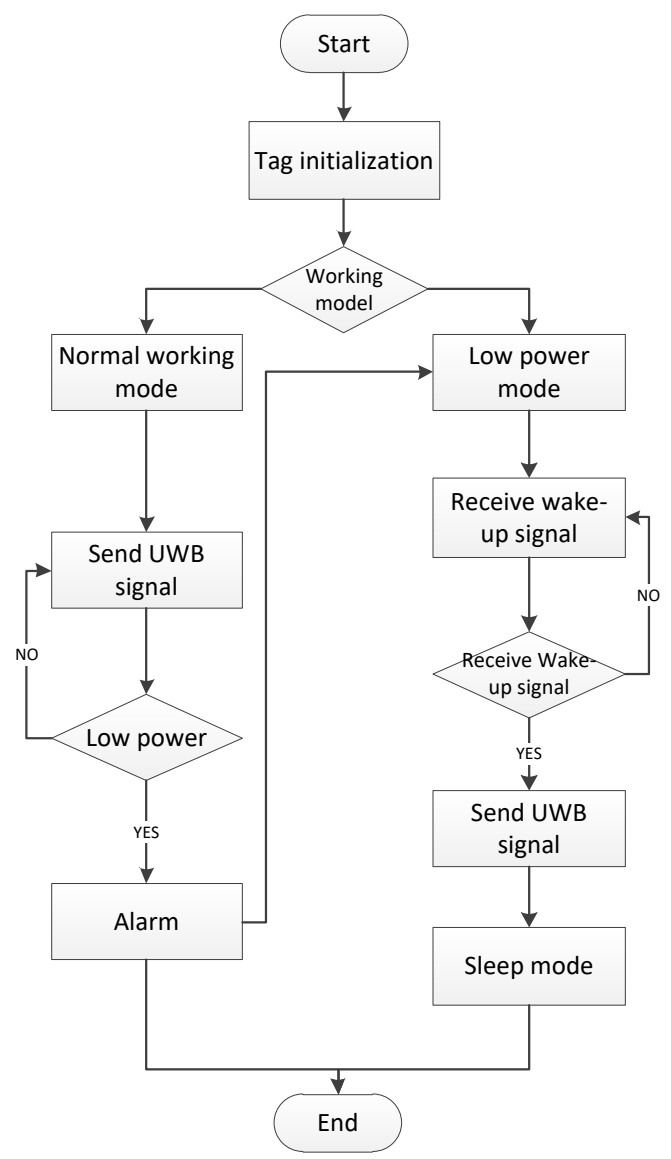

Fig. 6. Workflow of the tag

\subsection{Platform features}

The UWB-WSN real-time monitoring platform of underground personnel and equipment has the following features.

1. Real-time positioning of personnel: When the operators wearing location, tags enter an underground tunnel, they can be sensed by the sensors located across the tunnel. The sensors will send the information on the operators to the location engine. Then, the engine will calculate the identity, location and current time of the operators, and display the information in the monitoring centre via $3 \mathrm{D}$ visualization. In this way, the managers can identify the position of the operators at any time.

2. Electronic fence: According to the specific conditions, different underground tunnels can be allocated to different areas and given different access rights. When an operator without the access right enters an area, the location tag will give off an 
audio-visual alarm, and the monitoring platform will display the alarm information in real-time.

3. Emergency alarm: The operator should press the alarm button after finding a danger during the operation. Then, the alarm information will be sent to the monitoring centre, and then the emergency plan will be activated in time to evacuate the relevant personnel.

4. Search and rescue: When the operator is trapped underground, the monitoring terminal will guide rescuers to the correct position according to the signals issued by the location tag. If the location tag is damaged, the monitoring system will provide the rescuers with the last location information of the operator.

\section{$5 \quad$ Experiments and Results Analysis}

This chapter attempts to validate the UWB-WSN platform through underground positioning experiments on 15 targets in a $500 \mathrm{~m} \times 500 \mathrm{~m}$ region. The computing speed was neglected because the effect of the algorithm on positioning can be offset by improving the server performance. To simulate the motion of underground personnel, the location information was recorded at the speeds of $0 \mathrm{~m} / \mathrm{s}, 5 \mathrm{~m} / \mathrm{s}$ and $10 \mathrm{~m} / \mathrm{s}$. WiFi, ZigBee, RFID and UWB were all adopted to locate the moving targets.

The positioning accuracy can be demonstrated by the distance between the predicted and the actual locations:

$$
E_{i}=\sqrt{\left(x_{i}-x_{i 0}\right)^{2}+\left(y_{i}-y_{i 0}\right)^{2}}
$$

Then, the mean accuracy of the moving targets can be obtained as:

$$
E=\frac{\sum_{i=1}^{n} E_{i}}{N}=\frac{\sqrt{\left(x_{i}-x_{i 0}\right)^{2}+\left(y_{i}-y_{i 0}\right)^{2}}}{N}
$$

The experimental results are listed in table 2 .

Table 2. Experimental results under different conditions

\begin{tabular}{|c|c|c|c|}
\hline & $\mathbf{0 m} / \mathbf{s}$ & $\mathbf{5 m} / \mathbf{s}$ & $\mathbf{1 0 m} / \mathbf{s}$ \\
\hline WiFi & $5 \mathrm{~m}$ & $8 \mathrm{~m}$ & $12 \mathrm{~m}$ \\
\hline ZigBee & $7 \mathrm{~m}$ & $11 \mathrm{~m}$ & $17 \mathrm{~m}$ \\
\hline RFID & $26 \mathrm{~m}$ & $32 \mathrm{~m}$ & $40 \mathrm{~m}$ \\
\hline UWB & $0.08 \mathrm{~m}$ & 0.12 & 0.15 \\
\hline
\end{tabular}

Comparing the results, it is clear that the UWB-WSN platform outperformed the other methods in accuracy. In the event of an accident, a high positioning accuracy 
means highly efficient rescue and great likelihood of survival. Thus, the proposed platform is very suitable for personnel positioning in complex tunnels underground.

\section{Conclusions}

To realize accurate and reliable positioning of underground moving targets, this paper introduces UWB technique and WSN into underground target positioning. Taking the TODA as the positioning algorithm, the author designed a UWB-WSN monitoring platform for underground operators and a location tag. The proposed platform was compared with several other short-range wireless positioning methods through experiments. The results show that the UWB-WSN platform achieved unparalleled precision, which is very suitable for real-time positioning of personnel and equipment underground. The research findings shed new light on the mitigation of personnel and property losses in coal mine accidents.

\section{$7 \quad$ References}

[1] Shi, X.H. (2013). TDOA-based wireless positioning method and its performance. Journal of Southeast University (Natural Science Edition), 2(43), 252-256. Https://doi.org/10.3969/ j.issn.1001-0505.2013.02.005

[2] Wang, Z., Guo, J. (2017). UWB Indoor Positioning System Based on Improved TDOA Algorithm. Microcontrollers \& Embedded Systems, 5, 33-36

[3] Caffery, J. (2000). A new approach to the geometry of TOA location. IEEE Vehicular Technology Conference, 4, 1943-1949. https://doi.org/10.1109/VETECF.2000.886153

[4] Oksar, I. (2014). A Bluetooth signal strength based indoor localization method. Inter-national Conference on Systems, 3, 251-254

[5] Ge, B., Li, H. (2011). The Research on Zig Bee-based Mine Safety Monitoring System. Electric Information and Control Engineering, International Conference, 1837-1840

[6] Ma, H., Huang, X. (2013). Research of Mine Ventilation Parameters Tester Based on ZigBee Technology. Advanced Materials Research, 2482(734), 2672-2675. https://doi.org/10.4028/ www.scientific.net/AMR.734-737.2672

[7] Cheng, J., Gao, D., Wang, J., Wen, D. (2013). Coal Mine Safety Monitoring System Based on ZigBee and GPRS. Applied Mechanics and Materials, 422(3), 215-220. https://doi.org/10.4028/www.scientific.net/AMM.422.215

[8] Warm, C.D., Hsueh, C.S. (2010). Non-line of Sight Error Mitigation in Ultra-wide band Ranging Systems Using Biased Kalman Filter. Journal of Signal Processing Sys-tems, 64(3), 389-400. https://doi.org/10.1007/s11265-010-0493-6

[9] Gustafsson, F., Gunnarsson, F. (2003). Positioning using time-difference of arrival measurements. IEEE International Conference on Acoustics, 6, 208-212. https://doi.org/10.1109/ ICASSP.2003.1201741

[10] Hou, G., Wu, S., Gou, M. (2014). Design of tag in UWB high precision location sys-tem. China integrated circuit, 1(2), 63-66.

[11] Li, Y., Yang, L. (2014). Wireless positioning technology based on UWB. Electronic Design Engineering, 8(22), 139-142

[12] Yang, F. (2014). Research and Implementation of Wireless Location Algorithm Based on UWB. Northeastern University, 8-22. 
[13] Chen, R., Yang, Y. (2008). Application Research of UWB positioning algorithm in coal mine. Industry and Mine Automation, 6, 6-9.

[14] Sui, X., Yang, G., Hao, S., Wang, C., Xu, A. (2016). Dynamic positioning method based on TDOA in underground mines using UWB ranging. Journal of Navigation and Positioning, 4(23), 10-16

[15] Zhang, H. (2015). Research and application of high precision positioning method in indoor based on UWB. Liaoning Technical University, 10-39.

[16] Banerjee, S., Ghosh, A., Mitra, S.K. (2017). A modified mathematical model for life-time enhancement in wireless sensor network. Mathematical Modelling of Engineer-ing Problems, 4(2), 84-90. https://doi.org/10.18280/mmep.040204

\section{$8 \quad$ Authors}

Weicai Xie often works as associate professor, College of Electrical \& Information Engineering, Hunan Institute of Engineering, Xiangtan 411101, China. He Mainly engaged in motor control.

Xiaofeng $\mathbf{L i}$ is a master graduate student at the College of Electrical \& Information Engineering, Hunan Institute of Engineering, Xiangtan 411101, China. He Mainly engaged in Micro network Technology.

Xi Long often works in Power China Zhongnan Engineering Co. Ltd, Changsha 410014, China. He Mainly engaged in power electronic technology.

Article submitted 16 May 2018. Published as resubmitted by the authors 29 November 2018. 